\title{
Multi-agent Linear Dynamical Systems. Analyzing the Consensus Problem
}

\author{
Maria Isabel García-Planas \\ Matemàtica Aplicada I, \\ Universitat Politècnica de Catalunya, \\ C. Minería 1, Esc. C, 1-3, 08038 Barcelona, Spain \\ (e-mail: maria.isabel.garcia@upc.edu)
}

\begin{abstract}
In this paper the consensus problem is considered for multi-agent systems having an independent agent and fixed topology.
\end{abstract}

Index Terms-Multi-agent systems, consensus, control.

\section{INTRODUCTION}

It is well known the great interest created in many research communities, the study of control multiagents system, and there are an amount of literature as for example [3], [8], [10], [12]. It is due to the multi-agents appear in different areas as for example in consensus problem of communication networks [9] or formation control of mobile robots [2].

Jinhuan Wang, Daizhan Cheng and Xiaoming $\mathrm{Hu}$ in [10] study the consensus problem in the case of multiagent systems in which all agents have an identical linear dynamics and it is an stable linear system. In [3] this result is generalized to the case where the dynamic of the agents are controllable.

In this paper multiagent systems consisting of $k+$ 1 agents with dynamics

$$
\begin{aligned}
\dot{x}^{0} & =A_{0} x^{0} \\
\dot{x}^{1} & =A_{1} x^{1}+B_{1} u^{1} \\
\vdots & \\
\dot{x}^{k} & =A_{k} x^{k}+B_{k} u^{k}
\end{aligned}
$$

are considered. Wei Ni and Daizhan Cheng in [7] analyze the case where $A_{0}=A_{1}=\ldots=A_{k}$ and $B_{1}=\ldots=B_{k}$ this particular case has practical scenarios as the flight of groups of birds. It is obvious that in this case the mechanic of the first system is independent of the others, then consensus under a fixed topology can be easily obtained and it follows from the motion of the first equation. This consensus problem is known as leader-following consensus problem ([7]. [4]).

\section{PRELIMINARIES}

\section{A. Algebraic Graph theory}

We consider a graph $\mathcal{G}=(\mathcal{V}, \mathcal{E})$ of order $k$ with the set of vertices $\mathcal{V}=\{1, \ldots, k\}$ and the set of edges $\mathcal{E}=\{(i, j) \mid i, j \in \mathcal{V}\} \subset \mathcal{V} \times \mathcal{V}$.

Given an edge $(i, j) i$ is called the parent node and $j$ is called the child node and $j$ is in the neighbor of $i$, concretely we define the neighbor of $i$ and we denote it by $\mathcal{N}_{i}$ to the set $\mathcal{N}_{i}=\{j \in \mathcal{V} \mid$ $(i, j) \in \mathcal{E}\}$.

The graph is called undirected if verifies that $(i, j) \in \mathcal{E}$ if and only if $(j, i) \in \mathcal{E}$. The graph is called connected if there exists a path between any two vertices, otherwise is called disconnected.

Associated to the graph we consider a matrix $G=$ $\left(g_{i j}\right)$ called (unweighted) adjacency matrix defined as follows $g_{i i}=0, g_{i j}=1$ if $(i, j) \in \mathcal{E}$, and $g_{i j}=0$ otherwise.

In a more general case we can consider a weighted adjacency matrix is $G=\left(g_{i j}\right)$ with $g_{i i}=$ $0, g_{i j}>0$ if $(i, j) \in \mathcal{E}$, and $g_{i j}=0$ otherwise).

The Laplacian matrix of the graph is

$$
\mathcal{L}=\left(l_{i j}\right)= \begin{cases}\left|\mathcal{N}_{i}\right| & \text { if } i=j \\ -1 & \text { if } j \in \mathcal{N}_{i} \\ 0 & \text { otherwise }\end{cases}
$$

Remark 2.1: i) If the graph is undirected then the matrix $\mathcal{L}$ is symmetric, then there exist an orthogonal matrix $P$ such that $P \mathcal{L} P^{t}=\mathcal{D}$.

ii) If the graph is undirected then 0 is an eigenvalue of $\mathcal{L}$ and $(1, \ldots, 1)^{t}$ is the associated eigenvector. 
iii) If the graph is undirected and connected the eigenvalue 0 is simple.

For more details about graph theory see [11].

\section{B. Kronecker product}

Remember that $A=\left(a_{i j}\right) \in M_{n \times m}(\mathbb{C})$ and $B=$ $\left(b_{i j}\right) \in M_{p \times q}(\mathbb{C})$ the Kronecker product (see [5] for more information) is defined as follows

Definition 2.1: Let $A=\left(a_{j}^{i}\right) \in M_{n \times m}(\mathbb{C})$ and $B \in M_{p \times q}(\mathbb{C})$ be two matrices, the Kronecker product of $A$ and $B$, write $A \otimes B$, is the matrix

$A \otimes B=\left(\begin{array}{cccc}a_{1}^{1} B & a_{2}^{1} B & \ldots & a_{m}^{1} B \\ a_{1}^{2} B & a_{2}^{2} B & \ldots & a_{m}^{2} B \\ \vdots & \vdots & & \vdots \\ a_{1}^{n} B & a_{2}^{n} B & \ldots & a_{m}^{n} B\end{array}\right) \in M_{n p \times m q}(\mathbb{C})$

Kronecker product verifies the following properties

1) $(A+B) \otimes C=(A \otimes C)+(B \otimes C)$

2) $A \otimes(B+C)=(A \otimes B)+(A \otimes C)$

3) $(A \otimes B) \otimes C=A \otimes(B \otimes C)$

4) $(A \otimes B)^{t}=A^{t} \otimes B^{t}$

5) If $A \in G l(n ; \mathbb{C})$ and $B \in G l(p ; \mathbb{C}))$, then $A \otimes$ $B \in G l(n p ; \mathbb{C}))$ and $(A \otimes B)^{-1}=$ $A^{-1} \otimes B^{-1}$

6) If the products $A C$ and $B D$ are possible, then $(A \otimes B)(C \otimes D)=(A C) \otimes(B D)$

Associated to the Kronecker product, can be defined the vectorizing operator that transforms any matrix $A$ into a column vector, by placing the columns in the matrix one after another,

Definition 2.2: Let $X=\left(x_{j}^{i}\right) \in M_{n \times m}(\mathbb{C})$ be a matrix, and we denote $x_{i}=\left(x_{i}^{1}, \ldots, x_{i}^{n}\right)^{t}$ for $1 \leq$ $i \leq m$ the $i$-th column of the matrix $X$. We define the vectorizing operator $v e c$, as

$$
\begin{aligned}
& \text { vec }: M_{n \times m}(\mathbb{C}) \longrightarrow M_{n m \times 1}(\mathbb{C}) \\
& X \longrightarrow\left(\begin{array}{c}
x_{1} \\
x_{2} \\
\vdots \\
x_{m}
\end{array}\right)
\end{aligned}
$$

Obviously, vec is an isomorphism.

\section{Dynamic of multi-agent having identical dynam- ical mode}

Let us consider a group of $k$ identical agents. The dynamic of each agent is given by the following linear dynamical systems

$$
\begin{aligned}
\dot{x}^{1} & =A x^{1}+B u^{1} \\
\vdots & \\
\dot{x}^{k} & =A x^{k}+B u^{k}
\end{aligned}
$$

$x^{i} \in \mathbb{R}^{n}, u^{i} \in \mathbb{R}^{m}, 1 \leq i \leq k$.

We consider the undirected graph $\mathcal{G}$ with

i) Vertex set: $V=\{1, \ldots, k\}$

ii) Edge set: $\mathcal{E}=\{(i, j) \mid i, j \in V\} \subset V \times V$

defining the communication topology among agents.

Definition 2.3: Consider the system 1. We say that the consensus is achieved using local information if there is a state feedback

$$
u^{i}=K \sum_{j \in \mathcal{N}_{i}}\left(x^{i}-x^{j}\right), 1 \leq i \leq k
$$

such that

$$
\lim _{t \rightarrow \infty}\left\|x^{i}-x^{j}\right\|=0,1 \leq i, j \leq k .
$$

For simplify we will write $z^{i}=\sum_{j \in \mathcal{N}_{i}}\left(x^{i}-x^{j}\right), 1 \leq$ $i \leq k$.

The closed-loop system obtained under this feedback is as follows

$$
\dot{\mathcal{X}}=\mathcal{A X}+\mathcal{B K} \mathcal{Z}
$$

where

$$
\begin{gathered}
\mathcal{X}=\left(\begin{array}{c}
x^{1} \\
\vdots \\
x^{k}
\end{array}\right), \quad \dot{\mathcal{X}}=\left(\begin{array}{c}
\dot{x}^{1} \\
\vdots \\
\dot{x}^{k}
\end{array}\right), \\
\mathcal{A}=\left(\begin{array}{ccc}
A & & \\
& \ddots & \\
& & A
\end{array}\right), \mathcal{B}=\left(\begin{array}{ccc}
B & & \\
& \ddots & \\
& & B
\end{array}\right), \\
\mathcal{K}=\left(\begin{array}{ccc}
K & & \\
& \ddots & \\
& & K
\end{array}\right), \mathcal{Z}=\left(\begin{array}{c}
\sum_{j \in \mathcal{N}_{1}} x^{1}-x^{j} \\
\vdots \\
\sum_{j \in \mathcal{N}_{k}} x^{k}-x^{j}
\end{array}\right) .
\end{gathered}
$$

Following this notation we can conclude the following 
Proposition 2.1 ([10]): The closed-loop system can be described as

$$
\dot{\mathcal{X}}=\left(\left(I_{k} \otimes A\right)+\left(I_{k} \otimes B K\right)\left(\mathcal{L} \otimes I_{n}\right)\right) \mathcal{X} .
$$

Taking into account that the graph is undirected, following remark 2.1 , we have that there exists an orthogonal matrix $P \in G l(k ; \mathbb{R})$ such that $P \mathcal{L} P^{t}=$ $D=\operatorname{diag}\left(\lambda_{1}, \ldots, \lambda_{k}\right),\left(\lambda_{1} \geq \ldots \geq \lambda_{k}\right)$.

Corollary 2.1: The closed-loop system can be described in terms of the matrices $A, B$, the feedback $K$ and the eigenvalues of $\mathcal{L}$ in the following manner

$$
\dot{\widehat{\mathcal{X}}}=\left(\begin{array}{ccc}
A+\lambda_{1} B K & & \\
& \ddots & \\
& & A+\lambda_{k} B K
\end{array}\right) \widehat{X} .
$$

Remark 2.2: $\lim _{t \rightarrow \infty}\left\|P x^{i}-P x^{j}\right\|=0$ if and only if $\lim _{t \rightarrow \infty}\left\|x^{i}-x^{j}\right\|=0$.

1) Consensus problem: It would seem that if the graph is connected the consensus problem would be solvable of there is a $K$ such that the system 2 is stabilized. But taking into account that $\lambda_{1}=0$ this system is only stabilized if $\dot{x}^{1}=A x^{1}$ is stable.

Suppose now, that the system $(A, B)$ is controllable, so there exist $\bar{K}$ such that the close loop system $\dot{x}=(A+B \bar{K}) x=\bar{A} x$ is asymptotically stable and we apply all results presented in $\S 3$ over the group of $k$ identical agents, where the dynamic of each agent is given by the following linear dynamical systems

$$
\begin{aligned}
\dot{x}^{1} & =\bar{A} x^{1}+B u^{1} \\
\vdots & \\
\dot{x}^{k} & =\bar{A} x^{k}+B u^{k}
\end{aligned}
$$

$x^{i} \in \mathbb{R}^{n}, u^{i} \in \mathbb{R}^{m}, 1 \leq i \leq k$.

Lemma 2.1 ([10]): Let $(A, B)$ be a controllable pair of matrices and we consider the set of $k$-linear systems

$$
\dot{x}^{i}=A x^{i}+\lambda_{i} B u^{i}, 1 \leq i \leq k
$$

with $\lambda_{i}>0$. Then, there exist a feedback $K$ which simultaneously assigns the eigenvalues of the systems as negative as possible.

More concretely, for any $M>0$, there exist $u^{i}=$ $K x^{i}$ for $1 \leq i \leq k$ such that

$$
\operatorname{Re} \sigma\left(A+\lambda_{i} B K\right)<-M, 1 \leq i \leq k .
$$

$\left(\sigma\left(A+\lambda_{i} B K\right)\right.$ denotes de spectrum of $A+\lambda_{i} B K$ for each $1 \leq i \leq k)$.

As a corollary we can consider the consensus problem.

Corollary 2.2 ([3]): We consider the system 1 with a connect adjacent topology. If $(A, B)$ is a controllable pair then, the consensus is achieved by means the feedback defined in 2.3 and a feedback $\bar{K}$ stabilizing $(A, B)$.

Proof: Taking into account that the adjacent topology is connected we have that $0=\lambda_{1}<\lambda_{2} \leq$ $\ldots \leq \lambda_{k}$ and $(1, \ldots, 1)^{t}=\mathbf{1}_{k}$ is the eigenvector corresponding to the simple eigenvalue $\lambda_{1}=0$.

On the other hand we can find $\bar{K}$ stabilizing $(A, B)$ and then we can find $K$ stabilizing the associate system 2 , then we find $\mathcal{Z}$ such that $\lim _{t \rightarrow \infty} \mathcal{Z}=0$.

Using $\mathcal{Z}=\left(\mathcal{L} \otimes I_{n}\right) \mathcal{X}$ we have that $\lim _{t \rightarrow \infty} \mathcal{X}=$ $\mathbf{1}_{k} \otimes v$ for some vector $v \in \mathbb{R}^{n}$ and the consensus is obtained.

\section{DYNAMIC OF MULTI-AGENT HAVING NO IDENTICAL DYNAMICAL MODE}

We consider now, a multi-agent where the dynamic of each agent is given by the following dynamical systems

$$
\begin{aligned}
\dot{x}^{1} & =A_{1} x^{1}+B_{1} u^{1} \\
\vdots & \\
\dot{x}^{k} & =A_{k} x^{k}+B_{k} u^{k}
\end{aligned}
$$

$x^{i} \in \mathbb{R}^{n}, u^{i} \in \mathbb{R}^{m}, 1 \leq i \leq k$. Where matrices $A_{i}$ and $B_{i}$ are not necessarily equal.

The communication topology among agents is defined by means the undirected graph $\mathcal{G}$ with

i) Vertex set: $V=\{1, \ldots, k\}$

ii) Edge set: $\mathcal{E}=\{(i, j) \mid i, j \in V\} \subset V \times V$. an in a similar way as before, we have the following.

Definition 3.1: Consider the system 4. We say that the consensus is achieved using local information if there exists a state feedback

$$
u^{i}=K_{i} \sum_{j \in \mathcal{N}_{i}}\left(x^{i}-x^{j}\right), 1 \leq i \leq k
$$

such that

$$
\lim _{t \rightarrow \infty}\left\|x^{i}-x^{j}\right\|=0,1 \leq i, j \leq k .
$$


For simplicity we define $z^{i}=\sum_{j \in \mathcal{N}_{i}}\left(x^{i}-x^{j}\right), 1 \leq$ $i \leq k$.

The closed-loop system obtained under this feedback is as follows

$$
\dot{\mathcal{X}}=\mathcal{A} \mathcal{X}+\mathcal{B K} \mathcal{Z}
$$

where

$$
\begin{gathered}
\mathcal{X}=\left(\begin{array}{c}
x^{1} \\
\vdots \\
x^{k}
\end{array}\right), \quad \dot{\mathcal{X}}=\left(\begin{array}{c}
\dot{x}^{1} \\
\vdots \\
\dot{x}^{k}
\end{array}\right) \\
\mathcal{A}=\left(\begin{array}{ccc}
A_{1} & & \\
& \ddots & \\
& & A_{k}
\end{array}\right), \mathcal{B}=\left(\begin{array}{ccc}
B_{1} & \\
& \ddots & \\
& & B_{k}
\end{array}\right) \\
\mathcal{K}=\left(\begin{array}{lll}
K_{1} & & \\
& \ddots & \\
& & K_{k}
\end{array}\right), \mathcal{Z}=\left(\begin{array}{c}
\sum_{j \in \mathcal{N}_{1}} x^{1}-x^{j} \\
\vdots \\
\sum_{j \in \mathcal{N}_{k}} x^{k}-x^{j}
\end{array}\right)
\end{gathered}
$$

Calling

$$
\begin{aligned}
& \overline{\mathcal{B K}}= \\
& \left(\begin{array}{ccc}
B_{1} & & \\
& \ddots & \\
& & B_{k}
\end{array}\right)\left(\begin{array}{ccc}
K_{1} & & \\
& \ddots & \\
& & K_{k}
\end{array}\right)= \\
& \left(\begin{array}{ccc}
B_{1} K_{1} & & \\
& \ddots & \\
& & B_{k} K_{k}
\end{array}\right)
\end{aligned}
$$

and observing that

$$
\mathcal{Z}=\left(\mathcal{L} \otimes I_{n}\right) \mathcal{X}
$$

we deduce the following proposition

Proposition 3.1 ([3]): The closed-loop system can be deduced in terms of matrices $\mathcal{A}, \mathcal{B}$ and $\mathcal{K}$ in the following manner.

$$
\dot{\mathcal{X}}=\left(\mathcal{A}+\overline{\mathcal{B K}}\left(\mathcal{L} \otimes I_{n}\right)\right) \mathcal{X}
$$

We are interested in $K_{i}$ such that the consensus is achieved.

Proposition 3.2 ([3]): We consider the system 4 which a connected adjacent topology. If the system 5 is stable the consensus problem has a solution.

\section{Dynamic of MUlti-Agent With a LeAder}

In this section we analyze the dynamic of multiagent with special agent with independent motion of all other agents

The dynamic of each agent is given by the following dynamical systems

$$
\begin{aligned}
\dot{x}^{0} & =A_{0} x^{0} \\
\dot{x}^{1} & =A_{1} x^{1}+B_{1} u^{1} \\
\vdots & \\
\dot{x}^{k} & =A_{k} x^{k}+B_{k} u^{k}
\end{aligned}
$$

$x^{i} \in \mathbb{R}^{n}, u^{i} \in \mathbb{R}^{m}, 1 \leq i \leq k$.

The communication topology among agents is defined by means the undirected graph $\mathcal{G}$ with

i) Vertex set: $V=\{0,1, \ldots, k\}$

ii) Edge set: $\mathcal{E}=\{(i, j) \mid i, j \in V\} \subset V \times V$. an in a similar way as before, we have the following. - Definition 4.1: Consider the system 6. We say that the consensus is achieved using local information if there exists a state feedback

$$
u^{i}=K_{i} \sum_{j \in \mathcal{N}_{i}}\left(x^{i}-x^{j}\right), 0 \leq i \leq k
$$

such that

$$
\lim _{t \rightarrow \infty}\left\|x^{i}-x^{0}\right\|=0,1 \leq i \leq k .
$$

For simplicity we define $z^{i}=\sum_{j \in \mathcal{N}_{i}}\left(x^{i}-x^{j}\right), 1 \leq$ $i \leq k$.

The closed-loop system obtained under this feedback is as follows

$$
\dot{\mathcal{X}}=\mathcal{A} \mathcal{X}+\mathcal{B K} \mathcal{Z}
$$

where

$$
\mathcal{X}=\left(\begin{array}{c}
x^{0} \\
x^{1} \\
\vdots \\
x^{k}
\end{array}\right), \quad \dot{\mathcal{X}}=\left(\begin{array}{c}
\dot{x}^{0} \\
\dot{x}^{1} \\
\vdots \\
\dot{x}^{k}
\end{array}\right)
$$

$$
\mathcal{A}=\left(\begin{array}{llll}
A_{0} & & & \\
& A_{1} & & \\
& & \ddots & \\
& & & A_{k}
\end{array}\right),
$$




$$
\begin{gathered}
\mathcal{B}=\left(\begin{array}{llll}
0 & & & \\
& B_{1} & & \\
& & \ddots & \\
& & & B_{k}
\end{array}\right) \\
\mathcal{K}=\left(\begin{array}{cccc}
K_{0} & & & \\
& K_{1} & & \\
& & \ddots & \\
& & & K_{k}
\end{array}\right), \\
\mathcal{Z}=\left(\begin{array}{c}
\sum_{j \in \mathcal{N}_{0}} x^{0}-x^{j} \\
\sum_{j \in \mathcal{N}_{1}} x^{1}-x^{j} \\
\\
\sum_{j \in \mathcal{N}_{k}} x^{k}-x^{j}
\end{array}\right) .
\end{gathered}
$$

Calling

$$
\begin{aligned}
& \overline{\mathcal{B K}}=
\end{aligned}
$$

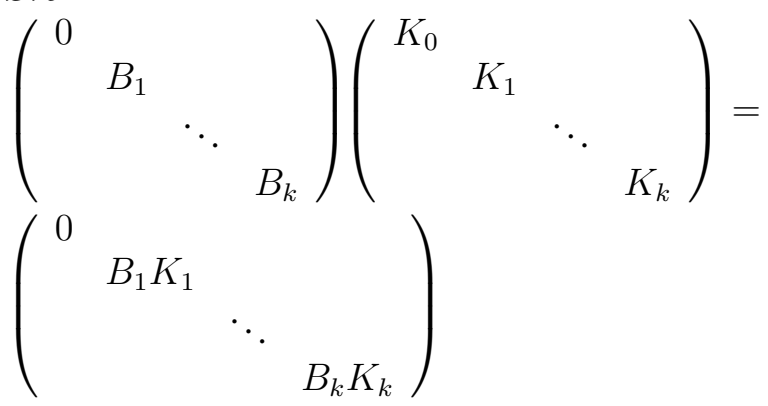

and observing that

$$
\mathcal{Z}=\left(\mathcal{L} \otimes I_{n}\right) \mathcal{X}
$$

we deduce the following proposition

Proposition 4.1: The closed-loop system can be deduced in terms of matrices $\mathcal{A}, \mathcal{B}$ and $\mathcal{K}$ in the following manner.

$$
\dot{\mathcal{X}}=\left(\mathcal{A}+\overline{\mathcal{B K}}\left(\mathcal{L} \otimes I_{n}\right)\right) \mathcal{X}
$$

We are interested in $K_{i}$ such that the consensus is achieved.

Proposition 4.2: We consider the system 6 which a connected adjacent topology. If the system 7 is asymptotically stable the consensus problem has a solution.

Proof: Remember that the zero state of a system $\dot{x}=A x$ is asymptotically stable if and only if all eigenvalues of $A$ have negative real parts (see [1]).

In our particular setup, and by hypothesis, all eigenvalues of the matrix $\mathbf{A}=\left(\mathcal{A}+\overline{\mathcal{B} K}\left(\mathcal{L} \otimes I_{n}\right)\right)$ have negative real parts. So, the solution of the system $\mathcal{X}=e^{\mathbf{A} t} \mathcal{X} \longrightarrow 0$ as $t \longrightarrow \infty$. But $\mathcal{X}=\left(\begin{array}{c}x^{0} \\ x^{1} \\ \vdots \\ x^{k}\end{array}\right)$. Then, the result is easily concluded.

Remark 4.1: If system 6 is stable, then the system $\dot{x}^{0}=A x^{0}$ is stable and the systems $\dot{x}^{i}=$ $A_{i} x^{i}+B_{i} u^{i}$, are stabilizable for all $1 \leq i \leq k$.

\section{A. Particular case}

We consider now the case where $A_{i}=A$ and $B_{i}=B$ for all $1 \leq i \leq k$ and for the first system also $A_{0}=A$ and we will try to obtain the matrix $\mathcal{K}$ with $K_{i}=k$ for all $1 \leq i \leq k$ such that the consensus is achieved.

Calling $y^{i}=x^{i}-x^{0}$, we have that $y^{i}-y^{j}=$ $x^{i}-x^{j}$ and $y^{0}=0$ and the multi-agent system can be rewritten as

$$
\begin{aligned}
\dot{x}^{0} & =A x^{0} \\
\dot{y}^{1} & =A y^{1}+B K \sum_{j \in \mathcal{N}_{1}}\left(y^{1}-y^{j}\right)= \\
& =A y^{1}+B K\left(\sum_{0 \neq j \in \mathcal{N}_{1}}\left(1+\alpha_{1}\right) y^{1}-y^{j}\right) \\
\vdots & \\
\dot{y}^{k} & =A y^{k}+B K \sum_{j \in \mathcal{N}_{k}}\left(y^{k}-y^{j}\right)= \\
& =A y^{k}+B K\left(\sum_{0 \neq j \in \mathcal{N}_{k}}\left(1+\alpha_{1}\right) y^{k}-y^{j}\right)
\end{aligned}
$$

where $\alpha_{i}=1$ if the agent $i$ is in the neighbor of the agent 0 , and $\alpha_{i}=0$ otherwise.

In this way we obtain a system in the variables $y$ as the systems of the subsection 1-C and we can apply the above results.

Notice that in this case, the matrix $\mathcal{L}_{y}$ coincides with the lower diagonal submatrix of order $k$ of the matrix $\mathcal{L}$ of the system (7) for our particular case. We can also observe that this matrix coincides with the Laplacian of the adjacency matrix of the graph obtained by considering only the relationship between agents $x^{i}$ regardless of the agent $x^{0}$ plus the diagonal matrix $\left(\alpha_{i}\right)$.

A similar result is obtained by Wei $\mathrm{Ni}$ and Daizhan Cheng in [7].

Example 4.1: We consider four agents with the following dynamics of each agent

$$
\begin{aligned}
& \dot{x}^{0}=A x^{0} \\
& \dot{x}^{1}=A x^{1}+B u^{1} \\
& \dot{x}^{2}=A x^{2}+B u^{2} \\
& \dot{x}^{3}=A x^{3}+B u^{3}
\end{aligned}
$$


The communication topology is defined by the graph $(\mathcal{V}, \mathcal{E})$ :

$$
V=\{0,1,2,3\}
$$

$\mathcal{E}=\{(i, j) \quad \mid \quad i, j \quad \in \quad V\}=$ $\{(0,1),(0,3),(1,2),(1,3)\} \subset V \times V$

and the adjacency matrix:

$$
G=\left(\begin{array}{llll}
0 & 1 & 0 & 1 \\
1 & 0 & 1 & 1 \\
0 & 1 & 0 & 0 \\
1 & 1 & 0 & 0
\end{array}\right)
$$

The neighbors of the parent nodes are $\mathcal{N}_{0}=$ $\{1,3\} \mathcal{N}_{1}=\{0,2,3\}, \mathcal{N}_{2}=\{1\}, \mathcal{N}_{3}=\{0,1\}$.

The Laplacian matrix of the graph is

$$
\mathcal{L}=\left(\begin{array}{cccc}
2 & -1 & 0 & -1 \\
-1 & 3 & -1 & -1 \\
0 & -1 & 1 & 0 \\
-1 & -1 & 0 & 2
\end{array}\right)
$$

Then the matrix $\mathcal{L}_{y}$ is

$$
\begin{aligned}
& \mathcal{L}_{y}=\left(\begin{array}{ccc}
3 & -1 & -1 \\
-1 & 1 & 0 \\
-1 & 0 & 2
\end{array}\right)= \\
& \left(\begin{array}{ccc}
2 & -1 & -1 \\
-1 & 1 & 0 \\
-1 & 0 & 1
\end{array}\right)+\left(\begin{array}{lll}
1 & & \\
& 0 & \\
& & 1
\end{array}\right) .
\end{aligned}
$$

\section{Conclusions}

In this paper the consensus problem is considered for multi-agent systems, in which all agents have an identical linear dynamic mode that can be of any order. A generalization to the case all agents are of the same order but do not have the same linear dynamic.

\section{REFERENCES}

[1] C.T. Chen, "Introduction to Linear System Theory". Holt, Rinehart and Winston Inc, New York, (1970).

[2] A. Fax, R. Murray, Information flow and cooperative control of vehicle formations, IEEE Trans. Automat. Control. 49, (9), pp. 1453-1464, (2004)

[3] M.I. García-Planas, Obtaining Consensus of Multi-agent Linear Dynamic Systems, Advances in Applied and Pure Mathematics, pp. 91-95, (2014)

[4] A. Jadbabaie, J. Lin, A.S. Morse, Coordination of groups of mobile autonomous agents using nearest neighbor rules, IEEE Transaction on Automatic Control. 48 (6), pp. 943-948, (2007).

[5] P. Lancaster, M. Tismenetsky, "The Thoery of Matrices". Academic Press. San Diego (1985).

[6] Z. Li, Z. Duan, G. Chen, Consensus of Multiagent Systems and Synchronization of Complex Networks: A unified Viewpoint, IEEE Trans. on Circuits and Systems, 57, (1), pp. 213-224, (2010).
[7] W. Ni, D. Cheng, Leader-following consensus of multi-agent systems under fixed switching topologies, Systems and Control Letters. 59, pp. 209-217, (2010).

[8] R.O. Saber, R.M. Murray, Consensus Protocols for Networks of Dynamic Agents, Report.

[9] R.O. Saber, R.M. Murray, Consensus problems in networks of agents with switching topology and time-delays, IEEE Trans. Automat. Control. 49, (9), pp. 1520-1533, (2004).

[10] J. Wang, D. Cheng, X. Hu, Consensus of multi-agent linear dynamics systems, Asian Journal of Control 10, (2), pp. 144155, (2008).

[11] D. West "Introduction to Graph Theory" Prentice Hall (3rd Edition), (2007).

[12] G. Xie, L. Wang, Consensus control for a class of networks of dynamic agents: switching topology, Proc. 2006 Amer. Contro. Conf., pp. 1382-1387, (2007). 